\title{
Neural afferents as potential targets to ameliorate FGF21-mediated sympathoexcitation
}

\author{
Kenichi Katsurada ${ }^{1,2} \cdot$ Kazuomi Kario $^{1}$
}

Received: 28 October 2021 / Revised: 2 November 2021 / Accepted: 2 November 2021 / Published online: 8 December 2021

(c) The Japanese Society of Hypertension 2021

The activation of the sympathetic nervous system is one of the hallmarks of cardiovascular diseases, including hypertension, heart failure, and chronic kidney disease (CKD), and sympathoexcitation is closely related to hemodynamic changes in these disease states. Fibroblast growth factor 21 (FGF21) is mainly secreted from the liver in response to metabolic stress to improve glucose and lipid metabolism and obesity. In addition to its metabolic effects, FGF21 also exerts cardiovascular effects and has been shown to be increased in the circulation of CKD patients. Circulating FGF21 can be elevated in response to impaired FGF21 sensitivity and reduced expression of the FGF21 receptor. This phenomenon is known as an FGF21 resistance state and contributes to metabolic dysfunction in diabetes and obesity. However, it is unknown to what degree elevated circulating FGF21 is involved in cardiovascular dysfunction in CKD.

In a study published in this issue of Hypertension Research [1], Matsui et al. investigated the associations among serum FGF21 levels, hemodynamics, and renal functions in humans. Brachial and aortic blood pressure (BP) and aortic pulse wave velocity (PWV) were measured to assess hemodynamics, and the estimated glomerular filtration rate (eGFR) and urinary albumin and creatinine ratio (UACR) were determined to assess renal function. The results showed that serum FGF21 levels were associated with systolic BP (SBP), pulse pressure (PP) and PWV. Subsequent mediation analyses demonstrated that serum FGF21 levels mediated the relationship of the eGFR with SBP, PP,

Kazuomi Kario

kkario@jichi.ac.jp

1 Division of Cardiovascular Medicine, Department of Internal Medicine, Jichi Medical University School of Medicine, Shimotsuke, Tochigi, Japan

2 Division of Clinical Pharmacology, Department of Pharmacology, Jichi Medical University School of Medicine, Shimotsuke, Tochigi, Japan and PWV and that of the UACR with PWV. These results suggest that FGF21 may increase aortic stiffness and blood pressure in patients with renal dysfunction.

Conflicting results on the effects of FGF21 on BP have been reported in animal studies. Intraperitoneal infusion of recombinant human FGF21 (rhFGF21) for 3 weeks reduced SBP in high-fructose-supplemented hypertensive rats; this reduced SBP was accompanied by improved baroreflex sensitivities, as assessed by responses to intravenous injection of phenylephrine and sodium nitroprusside [2]. In that study, acute intravenous injection of rhFGF21 did not exert any significant change in BP in either high-fructosesupplemented hypertensive rats or normotensive control rats. Another study showed that acute injection of rhFGF21 into the nodose ganglion, which transmits sensory vagal afferent signals from the visceral organs/tissues to the central nervous system, reduced BP and enhanced baroreflex sensitivity in normotensive Sprague-Dawley rats [3]. A previous report by some of the same authors demonstrated that mice with CKD induced by uninephrectomy and a high-phosphate diet exhibited hypertension during physical activity and augmented sympathetic nerve activity as assessed by power spectral analysis; importantly, the knockout of FGF21 restored these CKD-related adverse changes [4]. These findings from previous studies suggest that FGF21 exerts different actions depending on the injection site, duration of administration, and type of animal model.

The authors hypothesize that FGF21 mediates CKDrelated hypertension and aortic stiffness and propose the underlying mechanisms by which FGF21 activates the sympathetic nervous system and the FGF21 resistance state causes metabolic dysregulation leading to atherosclerosis. One way that FGF21 acts on the sympathetic nervous system is by entering the brain through the blood-brain barrier (BBB). Indeed, intracerebroventricular injection of FGF21 induces the expression of c-Fos, a marker of neuronal activation, in the suprachiasmatic nucleus, paraventricular nucleus (PVN), dorsomedial hypothalamus, and nucleus tractus solitarius (NTS) [5]. The PVN and NTS are 


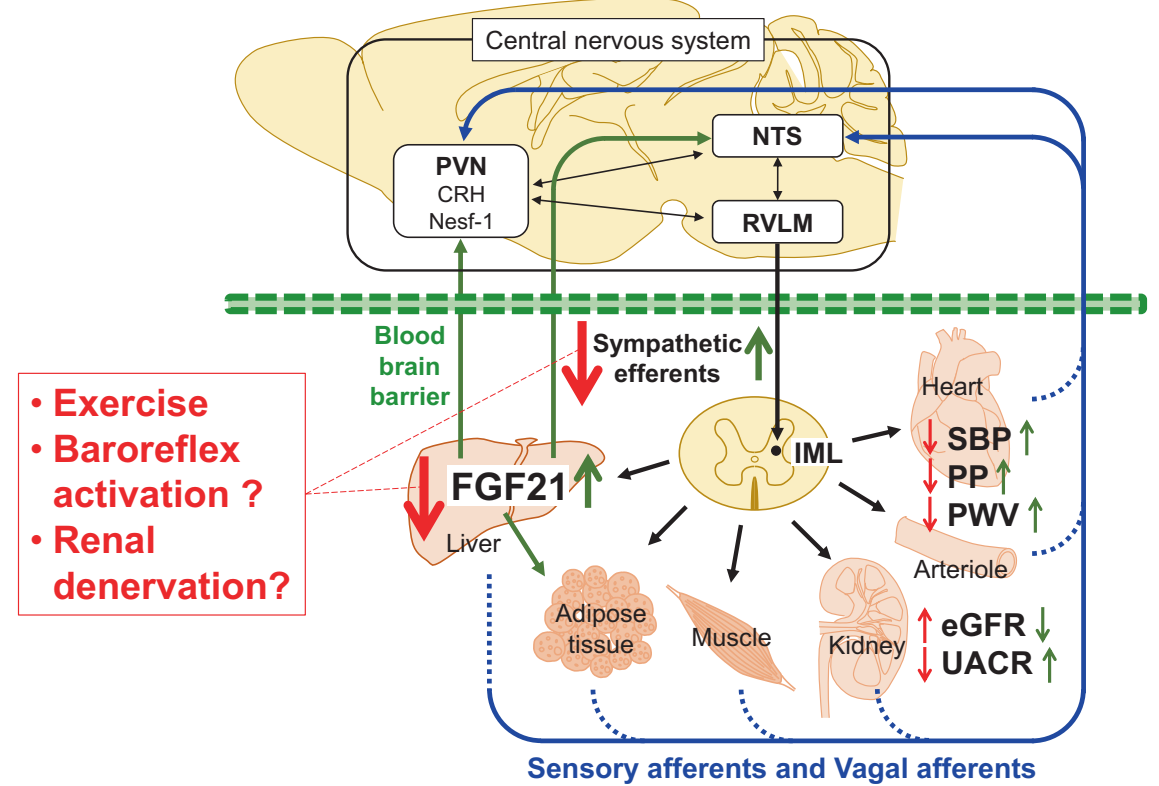

Fig. 1 Proposed model for the roles of FGF21 in regulating the sympathetic nervous system in chronic kidney disease. Green arrows show the potential sites targeted by FGF21 and the effects of FGF21. Black arrows show the interaction among the PVN, NTS and RVLM in the central nervous system and the sympathetic efferent pathways. Blue arrows show the sensory afferents and sensory vagal afferents. Red arrows show the possible effects of exercise, baroreflex activation

well-known cardiovascular centers interacting with each other and interacting with the rostral ventrolateral medulla (RVLM), which projects to the intermediolateral cell column (IML) in the spinal cord to determine sympathetic outflow to the periphery [6]. FGF21 activates PVN NUCB2/Nesfatin-1 neurons [5] and corticotropin-releasing hormone (CRH) neurons [7]. Both NUCB2/Nesfatin-1 and $\mathrm{CRH}$ neurons have been shown to increase sympathetic nerve activity.

FGF21 is suspected to also be involved in afferent neural pathways modulating the sympathetic nervous system. Afferent neural pathways, which are composed of sensory vagal afferents and sensory afferents, are another important way the sympathetic nervous system is regulated. These afferent neural pathways include the inhibitory or excitatory renal reflex, baroreflex, adipose afferent reflex, cardiac sympathetic afferent reflex, exercise pressor reflex, etc. Chronic sympathoexcitation is the pathological property of cardiovascular diseases such as hypertension, CKD, and heart failure. Resting afferent renal nerve activity is increased in deoxycorticosterone acetate-salt hypertensive rats [8], 2-kidney 1-clip CKD mice [9], and rats with heart failure induced by coronary artery ligation [10]. Selective afferent renal denervation performed by capsaicin application improves hypertension [8], CKD [9, 11] and heart failure [12] and mitigates sympathetic nerve overactivity as and renal denervation. $P V N$ paraventricular nucleus, NTS nucleus tractus solitarius, $R V L M$ rostral ventrolateral medulla, IML intermediolateral cell column, $C R H$ corticotropin-releasing hormone, Nesf1 nesfatin-1, FGF21 fibroblast growth factor 21, SBP systolic blood pressure, $P P$ pulse pressure, $P W V$ pulse wave velocity, $e G F R$ estimated glomerular filtration rate, $U A C R$ urinary albumin creatine ratio

assessed by direct recording of renal, splanchnic and lumbar sympathetic nerve activities and serum/urinary norepinephrine levels $[11,12]$. Afferent renal nerve signals are centrally integrated at the level of the PVN [6]. The expression of nitric oxide (NO) and neuronal NO synthase (nNOS) has been shown to be decreased in the PVN in various models of hypertension and heart failure. Selective afferent renal denervation restores the expression of $\mathrm{NO}$ and nNOS in the PVN, with concomitant sympathoinhibition, in rats with heart failure [12].

It is well known that signals from the baroreceptor in the carotid sinus and aortic arch are conveyed to the NTS in the brainstem and are relayed to the RVLM via the caudal ventrolateral medulla to maintain $\mathrm{BP}$ and heart rate (HR) and that together they comprise the baroreflex pathway [6].

The adipose afferent reflex (AAR) is a sympathoexcitatory reflex induced by the response of adipose tissue to sensory stimulations [13], resulting in increased energy expenditure. The AAR has a pivotal role in metabolic homeostasis and has hemodynamic effects. Sensory afferents from adipose tissue convey signals to the PVN and RVLM through the dorsal root ganglion and dorsal horn in the spinal cord. The AAR has been shown to increase BP and renal sympathetic nerve activity and can be initiated by leptin, adenosine, bradykinin, protons, or capsaicin [13]. In addition to these substrates, FGF21 could be involved in the 
AAR to activate sympathetic outflow, thereby affecting BP and arterial stiffness.

The cardiac sympathetic afferent reflex is also implicated in the maintenance of BP and HR. Afferent signals from the epicardium and myocardium are conveyed to the NTS in the brainstem, which interacts with the PVN and RVLM.

The exercise pressor reflex (EPR) is the mechanism by which sympathetic nerves are activated to increase BP and HR during exercise. EPR is evoked by muscle afferents that sense changes in contracting skeletal muscle and transmit information to the central nervous system to regulate BP and HR responses to exercise $[14,15]$.

The major sites in the central nervous system for afferent signal integration are the PVN and NTS, which dictate sympathetic outflow and regulate cardiovascular function. FGF21 can act on the PVN and NTS not only by penetrating the BBB but also through afferent pathways (Fig. 1). In the CKD condition, the excessive activation of renal afferents and possibly other sensory afferents changes the properties of the PVN and NTS, including the brain reninangiotensin system, NO/NOS and reactive oxygen species mechanisms, and the inflammation and immunity system [6]. These changes in the central nervous system might aggravate FGF21-mediated sympathoexcitation and contribute to the FGF21 resistance state and higher circulating FGF21 levels. In this issue, Matsui et al. suggest that exercise is one of the potential strategies to reduce elevated levels of circulating FGF21. It was previously reported that exercise training affects sensory afferents to improve sympathoexcitation in pathophysiological states [16]. These findings prompt us to speculate that exercise training acts on neural afferents to restore CKD-related adverse changes in the central nervous system, alleviating the FGF21 resistance state and reducing the levels of circulating FGF21 (Fig. 1). From this point of view, other neuromodulation therapies, such as baroreceptor activation therapy and renal denervation, are potential candidates to reduce the levels of circulating FGF21 concomitant with sympathoinhibition. It is worth investigating whether the levels of circulating FGF21 are changed after these neuromodulation therapies in CKD.

In conclusion, the current paper by Matsui et al. [1] demonstrated a potentially unfavorable mediation by FGF21 in elevating blood pressure and aortic stiffness with renal dysfunction in adult humans. The overactivation of the neural circuitry of sensory afferents and sympathetic efferents in CKD probably aggravates FGF21-mediated sympathoexcitation and contributes to the FGF21 resistance state. Intervention in this vicious neural circuit could be a potential strategy to improve the FGF21-mediated sympathoexcitation and FGF21 resistance state, reduce the levels of circulating FGF21 and suppress cardiovascular events in CKD patients (Fig. 1). Further basic and clinical studies are needed to explore the potential roles of FGF21 in regulating the cardiovascular system via afferent neural pathways in hypertension, heart failure, and CKD.

\section{Compliance with ethical standards}

Conflict of interest The authors declare no competing interests.

Publisher's note Springer Nature remains neutral with regard to jurisdictional claims in published maps and institutional affiliations.

\section{References}

1. Matsui M, Kosaki K, Kuro-o M, Saito C, Yamagata K, Maeda S. Circulating fibroblast growth factor 21 links hemodynamics with kidney function in middle-aged and older adults: a mediation analysis. Hypertens Res. 2021;2021-0222.R2. https://doi.org/10. 1038/s41440-021-00782-3.

2. He JL, Zhao M, Xia JJ, Guan J, Liu Y, Wang LQ, et al. FGF21 ameliorates the neurocontrol of blood pressure in the high fructose-drinking rats. Sci Rep. 2016;6:29582.

3. Chen P, Xu B, Feng Y, Li KX, Liu Z, Sun X, et al. FGF-21 ameliorates essential hypertension of SHR via baroreflex afferent function. Brain Res Bull. 2020;154:9-20.

4. Nakano T, Shiizaki K, Miura Y, Matsui M, Kosaki K, Mori S, et al. Increased fibroblast growth factor- 21 in chronic kidney disease is a trade-off between survival benefit and blood pressure dysregulation. Sci Rep. 2019;9:19247.

5. Santoso P, Nakata M, Shiizaki K, Boyang Z, Parmila K, Otgon-Uul $\mathrm{Z}$, et al. Fibroblast growth factor 21, assisted by elevated glucose, activates paraventricular nucleus NUCB2/Nesfatin-1 neurons to produce satiety under fed states. Sci Rep. 2017;7:45819.

6. Katsurada K, Ogoyama Y, Imai Y, Patel KP, Kario K. Renal denervation based on experimental rationale. Hypertens Res. 2021;44:1385-94.

7. Owen BM, Ding X, Morgan DA, Coate KC, Bookout AL, Rahmouni K, et al. FGF21 acts centrally to induce sympathetic nerve activity, energy expenditure, and weight loss. Cell Metab. 2014;20:670-7.

8. Banek CT, Knuepfer MM, Foss JD, Fiege JK, Asirvatham-Jeyaraj $\mathrm{N}$, Van Helden D, et al. Resting afferent renal nerve discharge and renal inflammation: elucidating the role of afferent and efferent renal nerves in deoxycorticosterone acetate salt hypertension. Hypertension 2016;68:1415-23.

9. Ong J, Kinsman BJ, Sved AF, Rush BM, Tan RJ, Carattino MD, et al. Renal sensory nerves increase sympathetic nerve activity and blood pressure in 2-kidney 1-clip hypertensive mice. J Neurophysiol. 2019;122:358-67.

10. Katsurada K, Nandi SS, Zheng H, Liu X, Sharma NM, Patel KP. GLP-1 mediated diuresis and natriuresis are blunted in heart failure and restored by selective afferent renal denervation. Cardiovascular Diabetol. 2020;19:57.

11. Veiga AC, Milanez MIO, Ferreira GR, Lopes NR, Santos CP, De Angelis $\mathrm{K}$, et al. Selective afferent renal denervation mitigates renal and splanchnic sympathetic nerve overactivity and renal function in chronic kidney disease-induced hypertension. J Hypertens. 2020;38:765-73.

12. Zheng H, Katsurada K, Liu X, Knuepfer MM, Patel KP. Specific afferent renal denervation prevents reduction in neuronal nitric oxide synthase within the paraventricular nucleus in rats with chronic heart failure. Hypertension 2018;72:667-75.

13. Dalmasso C, Leachman JR, Osborn JL, Loria AS. Sensory signals mediating high blood pressure via sympathetic activation: role of 
adipose afferent reflex. Am J Physiol Regul Integr Comp Physiol. 2020;318:R379-89.

14. Smith SA, Williams MA, Leal AK, Mitchell JH, Garry MG. Exercise pressor reflex function is altered in spontaneously hypertensive rats. J Physiol. 2006;577:1009-20.
15. Katsurada K, Kario K. Sympathetic modulation by antihypertensive drugs. J Clin Hypertens (Greenwich). 2021;23:1715-7.

16. Patel KP, Zheng H. Central neural control of sympathetic nerve activity in heart failure following exercise training. Am J Physiol Heart Circ Physiol. 2012;302:H527-37. 